\title{
EVITANDO MONSTRUOS: ANÁLISIS COMPARATIVO DE LAS NORMAS SOBRE SOCIEDADES EXTRANJERAS CONTROLADAS
}

\author{
Hugo Osorio Morales*
}

\begin{abstract}
RESUMEN
El trabajo analiza y compara el régimen jurídico-tributario aplicable a las Sociedades Extranjeras Controladas en Estados Unidos y Nueva Zelanda. En el diseño de esta normativa, se postula un enfoque cuidadoso y realista, considerando tanto su complejidad intrínseca como su potencial impacto económico. Tal enfoque es particularmente relevante para países que, como Chile, se encuentran en vías de desarrollo, debido a los limitados recursos disponibles para aplicar y fiscalizar estas normas y a la reticencia a afectar los flujos internacionales de inversión. Tres aspectos fueron seleccionados para su análisis comparado: la definición de Sociedad Extranjera Controlada, la Excepción de Entidad de Ingresos Activos y el tratamiento de los Pagos a Partes Relacionadas.
\end{abstract}

\section{SOCIEDADES EXTRANJERAS CONTROLADAS - ESTADOS UNIDOS DE AMÉRICA - NUEVA ZELANDA}

\section{Avoiding monsters. Comparative analysis of the regulations regarding controlled foreign companies}

\begin{abstract}
The controlled foreign companies' rules of the United States and New Zealand are analysed and compared. It is proposed that, considering the complexity of the matter and several particular elements that are discussed, a cautious and realistic approach in the design of the rules is advisable. Such approach is even more pertinent in the case of developing countries like Chile, due to their limited enforcement resources and natural reluctance to affect international investment. Three particular aspects are selected for the analysis: the controlled foreign companies' definition, the active entity test and the related parties' payments treatment.
\end{abstract}

\section{CONTROLLED FOREIGN COMPANIES - UNITED STATES OF AMERICA -} NEW ZEALAND

\footnotetext{
* Abogado, Magíster en Derecho, Profesor de Tributación de Empresas de la Universidad Austral de Chile, Jefe Departamento Jurídico Regional del Servicio de Impuestos Internos. Casilla 152, Valdivia, Chile, hugo.osorio.morales@gmail.com

Artículo recibido el 30 de agosto de 2010 y aceptado para su publicación por el Comité Editorial el 27 de octubre de 2010.
} 


\section{INTRODUCCIÓN}

$\mathrm{E}$ n el complejo campo tributario ${ }^{1}$, las normas relacionadas con las Sociedades Extranjeras Controladas (SECs) ${ }^{2}$ tienen el dudoso mérito de ser consideradas unánimemente una de las materias más difíciles y exigentes ${ }^{3}$. Muchos factores dan cuenta de ello. Primero, los sistemas que regulan las SECs deben prestar atención a una serie de políticas que usualmente se encuentran en conflicto ${ }^{4}$ : la neutralidad en exportación de capital, que tiende a eliminar o limitar los aplazamientos tributarios 5 con el objeto de lograr neutralidad en las decisiones de los contribuyentes nacionales sobre si invertir internamente o en el exterior ${ }^{6}$; la neutralidad en importación de capital, que tiende a permitir el aplazamiento tributario, facilitando que las compañías puedan competir internacionalmente ${ }^{7}$, incluso al costo de subsidiarlas con recursos de los contribuyentes locales ${ }^{8}$; el costo del cumplimiento tributario, el costo de fiscalización y la protección de la base tributaria interna, entre otros. En el esfuerzo de satisfacer todas estas políticas públicas, las normativas sobre SECs introducen matices, calificaciones y distinciones que inevitablemente llevan a niveles progresivamente mayores de complejidad. Segundo, el campo tributario se encuentra en esta materia en cambio permanente. Los aplazamientos tributarios en sí mismos son debatibles ${ }^{9}$, surgiendo reiteradamente la

${ }^{1}$ Para una discusión sobre las causas de tal complejidad, véase Jones, J.A. 'Tax Law: Rules or Principles?' en British Tax Review, 6, 1996, p. 580.

${ }^{2}$ Se ha traducido de esta forma el concepto y el acrónimo de Controlled Foreign Companies y CFC.

${ }^{3}$ Véase, por ejemplo, Burns, L., Controlled Foreign Companies: Taxation of Foreign Source Income, Longman Professional, Melbourne, 1992, p. xi; Arnold, B.J., The Taxation of Controlled Foreign Corporations: an International Comparison, Canadian Tax Foundation, Toronto, 1986, p. 516; McDaniel, P.R, Ault, J.A. y Repetti, J.R., Introduction to United States International Taxation (Quinta edición), Kluwer Law International, La Haya, 2005, p. 114.

${ }^{4}$ Para un análisis general sobre políticas tributarias en relación con las Sociedades Extranjeras Controladas, véase Arnold B.J., op. cit., pp. 52-63.

5 Se ha traducido como "Aplazamiento Tributario" el concepto anglosajón de deferral. Consiste en la posibilidad de un grupo económico nacional, con presencia en el extranjero, de postergar el pago de impuestos nacionales, por las utilidades generadas en el extranjero, hasta cuando tales utilidades sean remesadas al país. En Chile semejante postergación parece natural, precisamente porque no contamos con normativa sobre SECs. Lo que hacen estas normas es adelantar tal tributación cuando las empresas en el extranjero son controladas por las nacionales y se cumplen otros requisitos adicionales. Estos requisitos apuntan, en general, a que se trate de sociedades con ingresos de carácter "pasivo". Cuando se cumplen los requisitos mencionados, esto es, cuando las rentas de la sociedad extranjera controlada por la nacional debe tributar como renta de esta última, se dice que esas rentas han sido "atribuidas" (Attributed) a la sociedad doméstica.

${ }^{6}$ McDaniel, P.R., Ault J.A. y Repetti J.R., op. cit., p. 126.

${ }^{7}$ Arnold, B.J., op. cit., p. 134.

${ }^{8}$ McDaniel, P.R., Ault J.A. y Repetti J.R., op.cit., pp. 126-127.

${ }^{9}$ Para un exhaustivo análisis sobre políticas tributarias en relación con el Aplazamiento Tributario, véase Arnold B.J., op. cit. pp. 83-109, 408-411. 
discusión sobre la conveniencia de su completa abolición; las Compañías Multinacionales $(\mathrm{CMN})$, defensoras naturales de la neutralidad en importación de capital, han aumentado su poder e influencia dramáticamente en los últimos 30 años ${ }^{10}$; el consenso sobre el valor (las ventajas y desventajas) de la inversión extranjera, un elemento esencial para definir cuánto aplazamiento tributario es aceptable ${ }^{11}$, varía y ciertamente ha aumentado en los últimos 30 años; las crisis fiscales demandan protección urgente para la base tributaria interna, etc. Por ende, casos como el de Australia y Nueva Zelanda ${ }^{12}$, que presentan cambios continuos y radicales de política tributaria en esta materia, no son excepcionales, sino la regla. Tercero, las normativas relacionadas con las SECs tienen el potencial de impactar seriamente la economía de los países involucrados. La experiencia neozelandesa es, nuevamente, ilustrativa. El sistema de las SECs neozelandés vigente desde 1988 y hasta el año en curso trató de proteger el principio de neutralidad en exportación de capital, limitando severamente los aplazamientos tributarios. Los efectos fueron devastadores: en un período muy breve, la mayoría de las compañías con intereses internacionales migraron a otros países, especialmente a Australia, y Nueva Zelanda se transformó en el único país que, durante los años de intensa globalización comprendidos entre 1990 y 2004, disminuyó su inversión directa hacia el extranjero como un porcentaje de su Producto Interno Bruto ${ }^{13}$.

Las normas relacionadas con las SECs presentan un desafío particular para los países que, como Chile, se encuentran en vías de desarrollo. Estos países no sólo tienen escasos recursos para fiscalizar materias tributarias complejas, sino que también son reacios -si bien no siempre lo reconocen abiertamente- a implementar medidas que puedan ahuyentar a la inversión extranjera, la que en el común de los casos representa una necesidad urgente para ellos ${ }^{14}$. Estos factores pueden llevar a experiencias nefastas, como ilustran las pasadas experiencias con las normas de Precios de Transferencia. Las normas sobre Precios de Transferencias, como las de SECs, involucran materias técnicas complejas asociadas a las relaciones internacionales, afectan a los intereses de las CMNs y tienen el potencial de impactar en la inversión extranjera y la economía en general. Con reticencia, varios países en desarrollo promulgaron o expandieron estas normas en los últimos 10 años, básicamente como respuesta al informe de 1998 de la Organización para la Cooperación y el Desarrollo Económicos (OCDE) sobre Competencia Tributaria

${ }^{10}$ Algunos teóricos políticos consideran que la influencia de la economía capitalista transnacional ha alcanzado una magnitud que amenaza o limita los elementos democráticos en la definición de políticas públicas. Se trataría de una situación novedosa que, entre otros elementos, requiere o propicia un nuevo modelo democrático, desde el tradicional liberal hacia uno deliberativo. Véase Dryzek, J.S., Deliberative Democracy and Beyond: Liberals, Critics, Contestations, Oxford University Press, Nueva York, 2002, pp. 17-29.

11 Arnold, B.J., op. cit., p. 102.

${ }^{12}$ Véase Ault, H.J. y Arnold, B.J., Comparative Income Taxation: A Structural Analysis (Tercera edición), Kluwer Law International, La Haya, 2010, pp. 484 ss.

13 Gobierno de Nueva Zelanda, New Zealand's International Tax Review: A direction for Change, 2006, pp. 8-12.

${ }^{14}$ Biastrocchi, E., 'The use and Interpretation of Tax Treaties in the emerging world: Theory and Implications', en British Tax Review, 4, 2008, p. 365. 
Dañina ${ }^{15}$. Sin embargo, no sólo no existían incentivos reales para utilizarlas, sino que dichos países carecían de medios humanos y materiales para aplicarlas, de manera que estas normas generalmente permanecieron inactivas. Sin embargo, cuando algunos de estos países sufrieron crisis fiscales, la necesidad urgente de moneda "dura" los llevó, en ciertos casos, a aplicar programas de fiscalización arbitrarios y abusivos ${ }^{16}$. El resultado final fue no sólo un sistema de Precios de Transferencia complejo, inviable e inactivo, sino que, además, se dañó la credibilidad de las Administraciones Tributarias. Por éstas y otras razones similares, la doctrina especializada ha aconsejado a los países en desarrollo que consideren alternativas más simples de control, como normas antielusión apropiadas ${ }^{17} \mathrm{y}$, más generalmente, que sus sistemas tributarios se basen principalmente en impuestos indirectos, los que son menos complejos en general y también desde una perspectiva internacional $^{18}$.

No obstante estos riesgos y costos, lo cierto es que, desde cierto nivel de desarrollo, todos los países necesitan contar con regímenes normativos sobre SECs. Los aplazamientos tributarios ilimitados presentan, a largo plazo, un incentivo poderoso para la elusión tributaria, especialmente a través de la planificación tributaria agresiva y compleja y el uso de paraísos impositivos, violando no sólo los principios de equidad y la neutralidad en exportación de capital, sino, además, aportándole un carácter ilusorio a los principios de tributación mundial que subyacen en los sistemas de la mayoría de estos países ${ }^{19}$, incluido Chile. En el largo plazo, un país sin este tipo de normas simplemente hace voluntario el pago de tributos para las empresas nacionales con presencia en el exterior. El reciente ingreso de Chile a la OCDE implica, además, que más temprano que tarde se deberá contar con este tipo de normas, pues, al igual que aquellas sobre Precios de Transferencia, constituyen elementos esenciales de las recomendaciones de política tributaria que este organismo impone a sus asociados ${ }^{20}$. En fin, y sin considerar cierta condescendencia en la recomendación de basar los sistemas tributarios de países en desarrollo en impuestos indirectos, lo cierto es que semejante sistema tiende a fijar niveles de desigualdad social incompatibles con las intuiciones éticas dominantes en las sociedades occidentales.

15 Ídem.

16 Ídem, p. 366.

${ }^{17}$ En particular, en relación con el fenómeno de la Fuga de Capitales, véase Vann, R.J., "International Aspects of Income Tax" en Thuronyi V. (editor), Tax Law Design and Drafting, International Monetary Fund, Washington, 1996, p. 760.

18 Ídem, p. 810.

${ }^{19}$ Arnold, J.A. y Dibout, P., 'Limits on the use of low-tax regimes by multinational business: current measures and emerging trends. General Report', en Cabiers de Droit Fiscal International, LXXXVIb, 2001, p. 39.

${ }^{20}$ Apenas es necesario recordar que la reciente reforma sobre secreto bancario impuesta en la ley 20.046 fue una respuesta a los requerimientos de la OCDE para ingresar a dicho organismo. El que se haya aceptado como cumplimiento una normativa con tal nivel de complejidad y limitados poderes para la administración tributaria, al punto de hacerla única entre países que se refieren a sí mismos como paraísos fiscales, demuestra el interés de la OCDE por respetar las estrategias de sus asociados, por excéntricas que resulten. 
La posición de este trabajo es que, considerando la compleja realidad mencionada, todos los países, y muy en especial aquellos en vías de desarrollo, deben adoptar en esta materia un enfoque que sea a un tiempo cauteloso y razonable, práctico y realista. Un diseño ambicioso, aunque en teoría sea correcto, puede terminar volviéndose excesivamente complejo e inoperable. Tres elementos son relevantes para este enfoque: conciencia comparada, conciencia de negocios y simpleza. Respecto a la conciencia comparada, las experiencias internacionales no sólo presentan lecciones provechosas y soluciones sensatas, sino, además, un límite objetivo: en el actual mundo globalizado, un sistema tributario, en particular en su vertiente internacional, que no esté mínimamente alineado con las tendencias generales, tiene el riesgo de desarrollar graves distorsiones económicas ${ }^{21}$. La conciencia de los negocios, junto con la creciente movilidad del capital, es un elemento particularmente sensible para los países en desarrollo, los cuales, en los hechos, deben competir para recibir inversión extranjera ${ }^{22}$. Por último, en lo que respecta a la simpleza, los costos de cumplimiento y las reales capacidades de fiscalización también imponen límites objetivos. Es cierto que, en materias tributarias, la complejidad es inevitable; y es cierto también que en estas materias "la simpleza tiene un costo"23, porque usualmente para lograrla se minan objetivos de políticas públicas. Pero obviar este elemento lleva simplemente a diseños inaplicables, caros e inútiles.

Este trabajo presenta un análisis comparado de la normativa sobre las SECs. Atendida la complejidad de la materia y a objeto de proporcionar una discusión en profundidad, nos hemos enfocado en tres elementos: la definición de las SECs, la Excepción de Entidad de Ingresos Activos ${ }^{24}$ (EEIA) y los Pagos a Partes Relacionadas. Estos elementos han sido seleccionados porque apuntan a la cuestión principal del estudio: cómo desarrollar normativas sobre SECs que sean, al mismo tiempo, simples para resultar operables, suficientemente conscientes de los negocios para encargarse de la materia sin destruir o "espantar" a los capitales y consistentes para ser relevantes.

Se han seleccionado las experiencias de EE.UU. y Nueva Zelanda por diversas razones. Primero, por sus realidades divergentes en cuanto al tamaño de sus economías.

${ }^{21}$ La reciente reforma de las normas sobre las CFC en Nueva Zelanda, por ejemplo, no fue consecuencia de un cambio en los objetivos teóricos buscados, desde la 'neutralidad en la exportación de capital' que aseguraba el régimen previo, a una 'neutralidad en la importación de capital' que podría proveer el nuevo, sino un reconocimiento de que el sistema previo, distinto a los generalmente utilizados en el derecho comparado, era en definitiva dañino para la economía del país. Al respecto, véase Gobierno de Nueva Zelanda, Explanatory Note, Taxation (International Taxation, Life Insurance, and Remedial Matters) Bill 2008 (NZ) p. 66-67.

${ }^{22}$ Grandes países en desarrollo tienen, hasta cierto punto, algunas opciones adicionales: Véase Biastrocchi, E., op. cit., p. 365.

${ }^{23}$ Ault, H.J., "Tax Simplification from a Comparative Point of View”, en Lindencrona, G., Lodin S. y Wiman B. (editores), International Studies in Taxation: Law and Economics, Kluwer Law International, La Haya, 1999, p. 44.

${ }^{24}$ Hemos traducido de esta forma el término y acrónimo de Active Income Entity Exception AIEE. Se trata de una excepción general a la aplicación de las normas de SEC. En general, el concepto es que si una empresa desarrolla actividades activas en el extranjero, se autoriza el aplazamiento tributario. La razón es que si esas actividades son activas, responden a razones de negocios reales y no a maniobras de elusión. 
Es interesante observar si los conceptos centrales de política tributaria discutidos en relación a las SECs están presentes en las normativas concretas, sin importar el tamaño de las economías. Segundo, por su diversa evolución. El sistema de Estados Unidos de América se ha ido desarrollando durante casi 50 años, variando según la "temperatura ideológica" imperante ${ }^{25}$, mientras que el neozelandés fue completamente reformado el presente año, siguiendo una reformulación doctrinaria y teórica orgánica. Finalmente, es interesante observar la experiencia de Nueva Zelanda, pues es evidente que su tamaño y nivel de desarrollo lo asemejan en alguna medida a Chile y podría constituir un punto de partida en el diseño de las normas sobre SECs que, en el futuro cercano, deberá dictar nuestro país.

\section{Definición DE SEC}

En esta sección, se analiza cuándo una compañía extranjera se considera bajo el control de los contribuyentes nacionales. La definición de sociedad o compañía y de su calidad de extranjera no serán abordadas aquí ${ }^{26}$.

\subsection{Estados Unidos de América}

Bajo el sistema tributario de Estados Unidos, la definición de las SECs sigue dos pasos o etapas sucesivos. Primero, para ser calificada como una SEC, la corporación extranjera debe ser poseída por propietarios estadounidenses ${ }^{27}$. Segundo, la propiedad debe traspasar un cierto umbral. Las normas norteamericanas pertinentes definen a un propietario estadounidense como una persona -natural o jurídica- estadounidense que sea dueña del " $10 \%$ o más del poder votante combinado de todas las clases de acciones con derecho a voto en dicha corporación extranjera" 28 . De esta forma, si ninguna persona estadounidense controla una parte superior o igual al $10 \%$ del poder votante de una corporación extranjera, ésta no será considerada una SEC, incluso aunque todo el poder

${ }^{25}$ Sabido es, por ejemplo, que mientras Kennedy intentó eliminar toda postergación tributaria, Reagan la amplió considerablemente.

${ }^{26}$ En este punto, el sistema tributario de Estados Unidos de América sigue un test formal: la compañía es doméstica o extranjera dependiendo del lugar donde es creada u organizada. El lugar donde es controlada o dirigida resulta irrelevante: US Internal Revenue Code (en adelante, IRC) s 7701 (a)(4)-(5). Bajo el sistema de Nueva Zelanda, en cambio, una compañía es extranjera cuando no es residente en el país: Income Tax Act 2007 (NZ) s YA 1; y la residencia en el extranjero es determinada bajo cualquiera de cuatro test de residencia: el lugar donde la compañía es creada, el lugar donde tiene sus oficinas principales, el lugar donde tiene su centro de control u organización y el lugar donde los directores ejercen control, aun si la decisión o decisiones se materializan fuera de Nueva Zelanda: Income Tax Act 2007 (NZ) s YD 2.

${ }^{27}$ La regla es la siguiente: the foreign company must be owned by "United Stated shareholders" En este documento se ha traducido shareholders como "propietarios". Como se verá enseguida, sin embargo, en este caso el concepto es más restringido que el equivalente castellano.

${ }^{28}$ IRC s 951 (b). 
votante esté en manos de personas estadounidenses. Para evitar abusos a través del uso de personas vinculadas y/o cadenas de sociedades, existen reglamentos sobre propiedad indirecta ${ }^{29}$ y atribución de acciones ${ }^{30}$. El segundo paso dice que, para ser considerado una SEC, los propietarios estadounidenses deben ser dueños de más del 50\% del "poder votante total combinado de todas las clases de acciones... con derecho a voto o... el valor total de las acciones" ${ }^{31}$. Caben en este punto cuatro observaciones. Primero, este criterio, en contraste con el anterior, considera no sólo el poder votante, sino también el valor de las acciones ${ }^{32}$. Segundo, existen las mismas reglas de protección que en el caos anterior: la propiedad puede ser directa o indirecta y se aplican reglas constructivas. Tercero, es suficiente alcanzar el umbral de propiedad "en cualquier día durante el año tributario" de la $\mathrm{SEC}^{33}$, aunque se definen reglas de tiempo diferentes para determinar cuándo opera la limitación de aplazamiento tributario ${ }^{34}$. Finalmente, el porcentaje de "más de $50 \%$ " se reduce a "más del 25\%" en el caso de ingresos de seguros de ciertas compañías 35 .

\subsection{Nueva Zelanda}

La compleja definición de las SECs es uno de los pocos elementos de la normativa neozelandesa que no fue cambiado en la reciente reforma ${ }^{36}$, probablemente porque la simplificación no fue uno de sus principios directrices y la reducción de los costos de cumplimiento -el más cercano- se logra a través de la EEIA, que se discutirá en la próxima sección ${ }^{37}$. Nueva Zelanda utiliza tres pruebas diferentes para determinar si una corporación extranjera es o no controlada: una prueba estricta de control de jure, una prueba objetiva de control de facto y una prueba subjetiva de control de facto ${ }^{38}$. Aunque estas reglas se proveen como alternativas, es razonable considerar la prueba

${ }^{29}$ IRC s 958 (a).

${ }^{30}$ IRC s 958 (b). En general, estas reglas coinciden con las del IRC s 318(a), con algunas modificaciones.

${ }^{31}$ IRC s $957($ a).

32 Este elemento fue agregado en 1986 con el objeto de evitar o superar múltiples controversias que se habían presentado sobre la materia. Para un análisis histórico sobre tales disputas, véase McDaniel, P.R, Ault, J.A. y Repetti, J.R., op. cit., p. 115.

33 IRC s 957 (a).

${ }^{34}$ Las reglas CFC operan cuando la compañía extranjera mantiene esa situación por 30 o más días durante cualquier año tributario.

35 IRC s 957 (b).

36 Algunos cambios menores de referencia fueron introducidos en Income Tax Act 2007 (NZ) ss EX 1(2)(a), EX 1(2)(b), y EX 5(5)(c).

${ }^{37}$ Los principios fueron: en tanto sea posible, permitir a las compañías realizar sus legítimas actividades, minimizar los costos de cumplimiento tributario y mantener el nivel de protección de la base tributaria doméstica. Véase, Gobierno de Nueva Zelanda, New Zealand's International Tax Review: Developing an active income exception for controlled foreign companies: An official's issues paper (2007), pp. 6-7.

${ }^{38}$ La terminología se ha tomado de Burns, L., op. cit., pp. 16-75. Aunque en esta obra el autor se refiere a las reglas australianas de CFC, las de Nueva Zelanda son, en este respecto, prácticamente idénticas. 
subjetiva de control de facto como subsidiaria de las otras dos, dado que sus principios, esencialmente inciertos, sólo deberían usarse en la ausencia de las primeras, algo más claras y precisas ${ }^{39}$.

Bajo la primera regla, una compañía extranjera es considerada SEC si cinco o menos residentes neozelandeses controlan más del $50 \%$ de los intereses de la compañía. Bajo la segunda, se considera SEC si solo un residente neozelandés tiene el control del $40 \%$ o más de los intereses de la compañía, a menos que una tercera persona, no-residente de Nueva Zelanda, también tenga el control de $40 \%$ o más de los intereses de la compañía, en la misma categoría. Bajo la tercera, se considera que se trata de una SEC si cinco o menos residentes de Nueva Zelanda controlan "el ejercicio del derecho a voto para las decisiones de los accionistas o tenedores de interés de la compañía y, como resultado, controlan los asuntos de la compañía ${ }^{40}$. Los intereses generalmente se miden al final de cada trimestre ${ }^{41}$. Si cualquiera de estos test es positivo, la compañía se considera una SEC durante ese período fiscal ${ }^{42}$.

Las primeras dos definiciones se basan en el "test de control de interés", un instrumento complejo. Básicamente ${ }^{43}$, considera cuatro categorías de interés de control directo: ser accionista en una compañía extranjera, tener derechos para la toma de decisiones de los accionistas o tenedores de interés de una compañía extranjera, tener derecho a recibir ingresos de una compañía extranjera y tener derecho a recibir distribuciones de los activos netos de la compañía ${ }^{44}$. A este control directo se le suma el control indirecto, además de los controles directos e indirectos de personas asociadas ${ }^{45}$. "Control indirecto" se refiere a situaciones en que existen intereses a través de una persona asociada u otra $\mathrm{SEC}^{46}$. Una "persona asociada" es una categoría tributaria en sí misma, que se desarrolla a través de diferentes “tests de asociación” relacionados con los intereses de la compañía, fondos de inversiones, relaciones familiares y otros ${ }^{47}$.

${ }^{39}$ A una similar conclusión arriba Burns, L., ídem., p.19. En tal caso, sin embargo, el fundamento se encuentra además, en el Explanatory Memorandum de la propuesta legislativa australiana.

${ }^{40}$ Income Tax Act 2007 (NZ) s EX 1 (1).

${ }^{41}$ Income Tax Act 2007 (NZ) s EX 26 (1). En el sistema cuatrimestral, algunas normas antielusión se introducen para evitar la manipulación de intereses al terminar el período: Income Tax Act 2007 (NZ) ss GB 9-16. Asimismo, una persona puede elegir no ser medida sobre una base cuatrimestral: Income Tax Act 2007 (NZ) s EX 26 (2)- (4).

${ }^{42}$ Income Tax Act 2007 (NZ) s EX 1 (3).

43 Para una detallada descripción del test de control, véase New Zealand Master Tax Guide, Wolters Kluwer Business, Auckland, 2010, pp. 1047-1049.

${ }^{44}$ Income Tax Act 2007 (NZ) s EX 2.

45 Income Tax Act 2007 (NZ) s EX 3.

46 Income Tax Act 2007 (NZ) s EX 7.

47 Antes de abril de 2009, en materias tributarias internacionales se utilizaban reglas especiales para definir el concepto de "personas asociadas". A partir de dicha fecha, un nuevo concepto es utilizado en todo el ámbito jurídico tributario neozelandés: Income Tax Act 2007 (NZ) ss YB 1-16. 
En la tercera definición de SEC se utiliza el concepto de "control". Aunque recientemente se abolió la disposición que definía el concepto de "control” ${ }^{4}$, dicha disposición finalmente indicaba que existía control cuando un grupo de personas "controlan la compañía por cualquier otro método" 49 . Por ende, en la actualidad, al igual que antes de la abolición de dicha disposición, el uso del test subjetivo de control de facto se abre a un nivel importante de incertidumbre, dado que la materia debe ser resuelta considerando las circunstancias de cada caso ${ }^{50-51}$.

\subsection{Análisis}

El sistema estadounidense utiliza un sistema de doble porcentaje. Primero, considera un $10 \%$ para definir al propietario estadounidense y después el $50 \%$ al definir el control. El razonamiento detrás de estas reglas es enfocar el régimen en donde existe control efectivo y propiedad concentrada en Estados Unidos ${ }^{52}$. El uso de estos umbrales numéricos objetivos tienen dos efectos naturales: certeza y fácil manipulación. En efecto, si bien es fácil saber cuándo una compañía extranjera es controlada bajo estos parámetros, también es fácil organizarse y planear por adelantado ciertas organizaciones societarias que eluden efectivamente la activación de la reglamentación de SECs ${ }^{53}$.

El sistema neozelandés muestra una realidad completamente diversa: un nivel alto de incertidumbre y complejidad, debido al uso de diferentes tipos de tests de control y al esfuerzo evidente de impedir la manipulación del sistema. El razonamiento tras esta mezcla de sistemas y tests es que los umbrales de propiedad o control numéricos siempre pueden ser eludidos a través de una estructuración o planificación tributaria cuidadosas y, por ende, "es necesario suplementar los test de control estrictos con pruebas alternativas basadas en el concepto económico de control" para que el sistema SEC sea efectivo ${ }^{54}$. El enfoque de Nueva Zelanda no sólo es complejo e incierto, sino que además es comparativamente anómalo ${ }^{55}$. Tal sistema se explica, además, por razones históricas: Nueva Zelanda y Australia siguieron en su primitivo sistema el mismo camino en esta

${ }^{48}$ Income Tax Act 2007 (NZ) s YC 1 NZ Act. Esta sección fue derogada mediante la misma ley que reformó las reglas sobre CFC.

49 Income Tax Act 2007 (NZ) s YC 1(1)(c).

${ }^{50}$ A una conclusión similar, en relación con las normas análogas del sistema australiano, arriba el profesor Burns, L., op. cit,. p. 55.

51 Normas adicionales proveen excepciones relacionadas con Entidades de Inversión de Portafolio (Portfolio Investment Entities): Income Tax Act 2007 (NZ) s EX 1(2).

52 Véase Ault H.J. y Arnold B.J., op. cit.. p. 479.

53 Para otros ejemplos, véase Ault, H.J. y Arnold, B.J., op. cit., p. 479; McDaniel, P.R., Ault, J.A. y Repetti, J.R., op. cit. p. 116; Isenbergh, J., International Taxation (Segunda Edición), Fundation Press, Nueva York, 2005, p. 196.

${ }^{54}$ Burns, L., op. cit., p. 18. El análisis, hecho en relación a las reglas australianas sobre CFC, resultan pertinentes para las australianas.

55 En un reporte de 2001, de los 16 países analizados, sólo dos complementaban el test de control de jure con uno de facto: Nueva Zelanda y Australia. Véase Arnold, J.A. y Dibout, P., op. cit., p. 42. 
materia $^{56}$, tratando de eliminar los aplazamientos tributarios casi por completo ${ }^{57}$. En ese contexto, el uso de una definición exhaustiva de las SECs parece lógico.

Como se puede observar, dos elementos primarios que se proponen en este trabajo para el diseño de la normativa de SECs entran en conflicto: la simplicidad, representada en este caso por el doble control numérico de los EE.UU. y rechazada por los distintos tipos de reglas que operan en Nueva Zelanda y la relevancia que, en el caso norteamericano, podría verse minada a través de la manipulación de porcentajes. Es interesante entonces considerar, primero, si el sistema neozelandés evita la manipulación y, segundo, si el sistema objetivo de Estados Unidos efectivamente convierte al sistema en irrelevante. Hasta cierto punto, la respuesta a la primera pregunta es negativa. Sin importar el uso de los varios tipos de reglas, todavía existen posibilidades de manipulación en el sistema de Nueva Zelanda. Por ejemplo, si dos compañías tienen el $50 \%$ de control del interés o si 6 o más personas tienen el control de los asuntos de la compañía ${ }^{58}$. Respecto a la segunda interrogante, la respuesta pareciera ser - de nuevo- negativa, aunque en este caso la evidencia es más indirecta: la presión permanente que ejercen las compañías estadounidenses, en general, y las CMN, en particular, para derogar el sistema actual. Pocos aspectos del sistema tributario de EE.UU. son tan debatidos y controversiales ${ }^{59}$. Se puede argumentar que un sistema irrelevante no recibiría tal grado de atención.

Una posible respuesta a las posibilidades de manipulación que quedan abiertas en el sistema de Nueva Zelanda podría ser promulgar nuevas reglas adicionales. Pero este camino, como demuestra en general la experiencia comparada en la lucha contra la elusión tributaria, sólo lleva a alzas masivas en complejidad tributaria, con resultados limitados ${ }^{60}$. Para bien o-probablemente- para mal, los contribuyentes son demasiado creativos y están demasiado motivados a evitar impuestos como para ser detenidos de esta forma. Una segunda posibilidad sería promulgar reglas simples, como las del sistema de EE.UU. Este enfoque inevitablemente da posibilidades de manipulación, pero si se suplementa con una interpretación jurídica que esté en línea con el propósito de la norma, los resultados pueden ser bastante razonables. Es cierto que esta solución requiere de jueces sofisticados, que se muestren activos en la búsqueda de ese propósito jurídico al interpretar la ley tributaria -cuestión a lo menos dudosa en el caso chileno-, pero esa es probablemente la realidad en todas las cuestiones de elusión tributaria y no sólo en la reglamentación referente a las SECs.

56 Ambos sistemas se desarrollaron en paralelo: el neozelandés comenzó en abril de 1988 (Income Tax Amendment Act 1988), y el australiano en julio de 1990 (Taxation Laws Amendment Act (Foreign Income) Act 1990).

57 Esa era la intención primitiva en Australia. Sin embargo, tras una intensa presión por parte de los representantes de la industria, se introdujo una excepción de ingresos activos. Véase Burns, L., op. cit., pp. 10-13, 94-95.

58 Simples vías de manipulación fueron de inmediato detectadas bajo las reglas análogas australianas. Véase Burns, L., op. cit., p. xii.

${ }^{59}$ McDaniel, P.R, Ault, J.A. y Repetti, J.R., op. cit., p. 2.

${ }^{60}$ Freedman, J., Loomer, G y Vella, J., 'Corporate Tax Risk and Tax Avoidance: New Approaches', en British Tax Review, 1, 2009, p. 101. 


\section{La Excepción de Entidad de Ingresos Activos (EEIA)}

Históricamente, el objetivo principal de la normativa sobre SECs ha sido el ingreso pasivo $^{61-62}$; por esa razón, la mayoría de los países con tal reglamentación ha mantenido algún tipo de excepción en la que el ingreso no es atribuido cuando su origen es un negocio genuinamente activo. Esta EEIA puede seguir dos caminos. Puede ser diseñada como una excepción de entidad, o sea, puede tratar de identificar qué entidades desarrollan negocios activos, permitiendo aplazamientos tributarios de todos sus ingresos, activos o pasivos; o puede estar diseñada como una excepción de transacciones, es decir, puede intentar identificar el ingreso activo, permitiendo aplazamientos de sólo ese tipo de ingreso, rechazándolo para los demás, aun cuando ambos provengan del mismo ente ${ }^{63}$. Es difícil determinar a priori qué sistema es mejor ${ }^{64}$, porque si bien el enfoque de transacciones puede ser considerado más preciso, es también potencialmente más difícil de administrar y fiscalizar ${ }^{65}$. Por tradición, ambos sistemas fueron estudiados y discutidos como alternativos, pero de hecho ambos pueden coexistir. Bajo este enfoque híbrido se aplican dos tests. Primero, bajo el test de entidad, si la compañía se considera primordialmente un negocio activo, todos sus ingresos podrían estar sujetos a aplazamiento tributario y ningún ingreso sería atribuido. Segundo, si la compañía no pasa o "aprueba" el test, cada unidad de ingreso será analizado y sólo aquellas unidades que se consideren ingreso pasivo quedan sujetas a atribución. Australia fue el primer país en utilizar este método híbrido ${ }^{66}$. El actual sistema neozelandés sigue el mismo enfoque.

En línea con la práctica general, ambos sistemas, el norteamericano y el de Nueva Zelanda, consideran la naturaleza del ingreso para definir cuándo éste es atribuido a accionistas o propietarios domésticos. Generalmente, en el caso de Nueva Zelanda, sólo el

${ }^{61}$ Arnold, J.A. y Dibout, P., op. cit., p. 51.

${ }^{62} \mathrm{La}$ distinción entre ingresos activos y pasivos es ajena nuestra tradición jurídica. Se trata de un área con altos niveles de complejidad y controversia que amerita por sí sola un estudio. En términos muy generales, la distinción apunta a distinguir entre actividades que se realizan en determinado país por las ventajas económicas comparativas reales que otorga, de aquellas que sólo se explican por las ventajas tributarias que se obtienen por hacerlo allí. Debe recordarse que, a fin de cuenta, las normas sobre SECs son normas contra la elusión tributaria. Si una compañía invierte en un paraíso fiscal, o en un país con un tratado de doble comercio con un paraíso fiscal, no porque los negocios que pretende hacer allí sean rentables, sino porque las ventajas tributarias lo justifican, tales ingresos serán calificados de pasivos. Debe tenerse cuidado, sin embargo, con el concepto de paraíso fiscal, porque si bien algunos países todavía utilizan "listas negras" de tales países, calificando de pasivos todos los ingresos que de allí provengan, es evidente que, por ejemplo la inversión en un Resort en Las Bahamas puede responder a razones de negocios reales y no a simple elusión tributaria. Remesas canalizadas a través de un paraíso fiscal, en cambio, provenientes de supermercados o fábricas en terceros países, difícilmente tienen otra justificación que evitar los impuestos domésticos.

63 Burns, L., op. cit., pp. 95-96.

${ }^{64}$ Para una discusión general sobre la materia, arribando a igualmente "no conclusivas" respuestas, véase Arnold, B.J., op. cit., pp. 437-444, 448-450.

${ }^{65}$ Explanatory Note, Taxation (International Taxation, Life Insurance, and Remedial Matters) Bill 2008 (NZ), p. 68.

${ }^{66}$ Burns L., op. cit., p. 96. 
ingreso pasivo se atribuye ${ }^{67}$, incluso si la SEC no pasa la EEIA ${ }^{68}$. En el caso de EE.UU., la situación es algo más compleja, pero sin duda hay un esfuerzo general a distinguir entre el ingreso pasivo y el ingreso legítimo de negocios, considerando sólo el primero como ingreso "Subsección F" 69 , sujeto a atribución 70 .

Este trabajo enfoca su interés en la EEIA porque, si es correctamente diseñada, tiene el potencial de reducir de manera significativa los costos de cumplimiento y fiscalización relacionados con las SECs. En consecuencia, las Administraciones Tributarias pueden centrar sus esfuerzos sólo en aquellas compañías que presentan riesgos sustanciales para la hacienda pública, o para la neutralidad en la exportación de capital. Este enfoque es también consistente con la tendencia actual en las Administraciones Tributarias en el mundo en los últimos 20 años, abandonando el objetivo -o la pretensión- de auditar todo riesgo, para enfocar sus esfuerzos sólo en ciertos espacios determinados de alto riesgo ${ }^{71}$. En fin, este interés parece en particular relevante para los países en desarrollo, los que sólo pueden fiscalizar una reglamentación sobre SECs diseñada de esta manera. Sin embargo, con certeza, la EEIA y el enfoque de entidades en general tienen el costo inherente de permitir aplazamientos tributarios, lo que, sujeto a abusos, puede resultar excesivo, erosionando la base tributaria interna y la política de neutralidad en la exportación de capital. Por eso, al diseñar las EEIA resulta esencial definir el nivel que activa las reglas de atribución y aquellas que controlan su abuso ${ }^{72}$.

\subsection{Estados Unidos de América}

El sistema sobre SECs norteamericano no contempla una EEIA general. Sin embargo, la excepción "de minimis", aunque más limitada, tiene un objetivo similar y efectos análogos. El sistema estadounidense también considera una regla "de maximus" peculiar y de difícil justificación ${ }^{73}$.

67 Véase Income Tax Act 2007 (NZ) s EX 20B- EX 21.

68 "Incluso si una CFC no pasa el test de negocios activos, sólo sus ingresos pasivos serán sujetos de impuesto en Nueva Zelanda":

Gobierno de Nueva Zelanda, New Zealand's International Tax Review: Developing an active income exception for controlled foreign companies: An official's issues paper (2007), p. 7.

69 Generalmente se habla de "ingreso Subsección F", pues esta es la sección de código Tributario norteamericano que regula a las SECs.

${ }^{70}$ Ault, H.J. y Arnold, B.J., op. cit., p. 478.

${ }^{71}$ Véase, por ejemplo, OECD, Forum Tax Administration Compliance Sub Group. Final Report, Monitoring Taxpayers' Compliance: A Practical Guide Based on Revenue Based Experience (2008); también, OECD, Tax Administration in OECD and Selected Non-OECD Countries: Comparative Information series (2008).

${ }^{72}$ Explanatory Note, Taxation (International Taxation, Life Insurance, and Remedial Matters) Bill 2008 (NZ) at 69

73 Arnold, B.J., op. cit., p. 396. 
Los ingresos "Subsección F" incluyen varios elementos ${ }^{74}$, siendo los ingresos de Compañía Instrumental Extranjera el más importante ${ }^{75}$ y que incluye, a su vez, varios elementos $^{76}$. Si el ingreso de Compañía Instrumental Extranjera y el ingreso por concepto de seguros -otro tipo dentro de la "Subsección F"- suman no más del 5\% del ingreso bruto ${ }^{77}$ o US $\$ 1.000 .000$, ninguna parte de ese ingreso será considerado como ingreso de Compañía Instrumental Extranjera o de seguros ${ }^{78}$. Ésta es la regla de minimis. En cambio, si la suma de ambos ingresos excede el $70 \%$ del ingreso bruto para el año tributario, todo el ingreso se considerará ingreso de Compañía Instrumental Extranjera o de seguros ${ }^{79}$. Esta es la regla de maximus. El resultado es que, entre los umbrales de $5 \%$ y $70 \%$ es necesario distinguir si el ingreso es parte o no de este tipo de ingresos y, en todos los otros casos, el ingreso completo es considerado uno u otro. En otras palabras, dentro de estos límites, no se aplica ningún test y cada ingreso debe definirse individualmente. La relativa irrelevancia del test en la actualidad no es inherente a éste en sí, sino una consecuencia de su evolución. Originalmente, no había límites numéricos y el porcentaje estaba establecido en $30 \%$. Sólo con posterioridad, bajo sucesivas reformas que tendían a limitar los aplazamientos tributarios, se introdujo el límite numérico y el porcentaje fue reducido al $10 \%{ }^{80}$, primero, y luego al presente $5 \%$. Esto significa que, en su origen, la excepción de minimis podía potencialmente cubrir a la mayoría de las SECs y que, considerando que ambos tipos de ingresos cubrían la mayoría del ingreso, esta regla operaba efectivamente como una EEIA.

Es interesante notar, además, que el sistema tributario norteamericano contaba antaño con una EEIA. En efecto, bajo la derogada reglamentación de Sociedades Personales Instrumentales Extranjeras ${ }^{81,82}$, existía tal excepción, dado que la definición de este tipo de compañías dependía de que se tuviera un cierto porcentaje - $50 \%$ generalmente, $60 \%$ el primer año- de ingresos de Compañía Instrumental Extranjera ${ }^{83}$ que era básicamente ingreso pasivo. Si el test era activado, todo el ingreso era atribuido a los propietarios estadounidenses ${ }^{84}$. Debido a que las reglas sobre SECs en la "Subsección F" fueron ex-

\footnotetext{
${ }^{74}$ IRC s 952.

75 Se ha traducido de esta forma el concepto utilizado en la legislación norteamericana de foreign-base company income. Se trata de un concepto que en general apunta a sumar a los ingresos directos de una compañía, aquellos que provienen de otras compañía bajo su control.

${ }^{76}$ IRC s 954.

77 Ingreso Bruto se encuentra definido en IRC s.954 (b) (C).

${ }^{78}$ IRC s 954 (b) (3) (A).

${ }^{79}$ IRC s 954 (b) (3) (B).

${ }^{80}$ Arnold, B.J., op. cit., p. 396.

${ }^{81}$ Se ha definido así el concepto de Foreign Personal Holding Companies.

${ }^{82}$ La ahora derogada s 551-558 IRC.

${ }^{83}$ Foreign-holding company income

${ }^{84}$ Arnold, B.J., op. cit., pp. 396, 381.
} 
pandiéndose en forma progresiva, la normativa de Sociedades Personales Instrumentales Extranjeras pasó a ser redundante hasta que, en 2004, fue finalmente derogada ${ }^{85,86}$.

\subsection{Nueva Zelanda}

En un marcado contraste con EE.UU., se pretende que la EEIA sea una pieza central de la novedosa normativa SEC de Nueva Zelanda. La intención general es clara: ningún ingreso será atribuido cuando las SEC desarrollen negocios "primariamente activos" La disposición es asimismo simple: una SEC activa no-atribuyente ${ }^{88}$, no atribuirá ingresos o pérdidas a sus propietarios ${ }^{89}$. En reglas generales, una SEC es una "SEC activa no-atribuyente" cuando su ingreso pasivo es menor al 5\%. A estas SECs, entonces, se les permite aplazamientos tributarios ilimitados, sin atribución alguna ${ }^{90}$.

Esta excepción y correspondiente test no sólo fueron diseñados como un portal que "filtre a los negocios primariamente activos fuera de las reglas de atribución"91, sino también como herramientas que simplificaran y abarataran el cumplimiento tributario. Como es natural, si para realizar este test se requirieran los mismos cálculos que el sistema general de atribución de las SECs, cualquier reducción en los gastos de cumplimiento sería ilusoria. Por ende, un sistema especial de cálculo para el test fue diseñado, buscando, por una parte, simpleza y reducción de $\operatorname{costos}^{92} \mathrm{y}$, por la otra, asegurar que la información utilizada fuera consistente ${ }^{93}$. La solución o el acuerdo al que se llegó fue ofrecer a los contribuyentes tres opciones básicas para rendir el test: bajo el sistema de cuentas financieras auditadas preparadas en cumplimiento con las Normas Internacionales de Información Financiera (IFRS) ${ }^{94}$, o bajo su equivalente neozelandés (NZ IFRS), o la reglamentación tributaria de Nueva Zelanda95. Este último es el test

${ }^{85}$ Isenbergh, J., op. cit., p. 196.

86 El actual sistema de Estados Unidos de América considera un muy limitado 'test de ingreso activo' (active income test) en el caso de compañías creadas en ciertos territorios: IRC s 957(c). Además, existen en dicho país numerosas normas que excluyen de la atribución (attribution) ingresos provenientes de negocios activos, pero tales normas no constituyen reales tests de ingreso activo, pues sólo son usados para determinar qué ingresos son o no atribuidos, no si las reglas de CFC deben ser aplicadas en primer lugar. Véase, por ejemplo: IRC s 954 (c)(2).

87 Gobierno de Nueva Zelanda, New Zealand's International Tax Review: Developing an active income exception for controlled foreign companies: An official's issues paper (2007), p. 11.

${ }^{88}$ Non-attributing active CFC.

${ }^{89}$ Income Tax Act 2007 (NZ) s EX 18A (2) (b) (3).

90 Bajo base financiera ajustada: Income Tax Act 2007 (NZ) s EX 21 E (3) (a); bajo base tributaria: Income Tax Act 2007 (NZ) s EX 21D (2) (a).

${ }^{91}$ Gobierno de Nueva Zelanda, New Zealand's International Tax Review: Developing an active income exception for controlled foreign companies: An official's issues paper (2007), p. 12.

92 Ídem, pp. 9, 12.

${ }^{9}$ Ídem, p. 12.

${ }^{94}$ International Financial Reporting Standards-IFRS.

${ }^{9}$ Ídem, p. 13. 
que se aplica por defecto ${ }^{96}$. Para facilitar la aplicación - y reducir los costos- del test por defecto, en él se requiere calcular sólo los ítems brutos del ingreso ${ }^{97}$, evitando de esta manera "otros pasos en el cálculo tributario completo, incluyendo la determinación de ítems de gastos deducibles"98.

En este punto, surgió una discusión interesante relacionada con los costos del test. Bajo el sistema previo, aunque se limitaban significativamente los aplazamientos tributarios, si una SEC residía en uno de los países de la 'lista gris'99, no se atribuía ingreso alguno a los dueños neozelandeses, sin necesidad de test alguno. Los nuevos tests reemplazaron esta lista y, en consecuencia, en todos los casos, el nuevo sistema implicaba costos adicionales para los tenedores de interés de SEC con residencia en estos países. El gobierno consideró que estos costos adicionales eran necesarios para proteger la integridad del sistema ${ }^{100}$, pero casi todas las observaciones presentadas por partes interesadas consideraban que el nuevo sistema no sólo era excesivo, en relación a los estándares requeridos, sino que además era injusto, porque afectaba en especial a los pequeños y medianos propietarios, los que de manera usual tenían intereses en sólo una SEC -habitualmente residente en Australia- mismos que, antes de la reforma, no debían rendir ningún test ${ }^{101}$. La ironía de la situación fue llamativa: las grandes compañías, principales beneficiarios del nuevo sistema, representaban al gobierno su falta de sensibilidad hacia los pequeños contribuyentes. La respuesta del gobierno fue, básicamente, mantener la lista gris, pero sólo en lo que respecta a Australia ${ }^{102}$, "porque muchas entidades pequeñas tienen SEC en Australia, e incluso la pequeña carga que les significa el 'test de negocio activo' podría ser excesiva para ellos" ${ }^{103}$. Esta solución de todas formas implicó un alza en los costos de cumplimiento de pequeñas SEC fuera de Australia. Sin embargo, la solución pareció resolver el problema, considerando que sólo un $20 \%$ de las SECs residían en un país de la lista gris que no fuera Australia ${ }^{104}$ y

\footnotetext{
${ }^{96}$ Income Tax Act 2007 (NZ) s EX 21D.

97 Income Tax Act 2007 (NZ) s EX 21D (4)-(9).

98 Gobierno de Nueva Zelanda, New Zealand's International Tax Review: Developing an active income exception for controlled foreign companies: An official's issues paper (2007), p. 15.

${ }^{99}$ Los países eran Australia, Canadá, Alemania, Japón, Gran Bretaña, los Estados Unidos de América y España: Income Tax Act 2007 (NZ) s EX 22 (substituted), s EX 23(repealed), YA 1 (substituted), Sch 24 (amended), todos de Taxation (International Taxation, Life Insurance, and Remedial Matters) Act 2009 (NZ).

${ }^{100}$ Gobierno de Nueva Zelanda, New Zealand's International Tax Review: Developing an active income exception for controlled foreign companies: An official's issues paper (2007), p. 13.

101 Véase por ejemplo, New Zealand Institute of Chartered Accountants, Submission on the Government Officials' issues paper: New Zealand's International Tax Review, Developing anactive income exemption for controlled foreign companies (2007); Business New Zealand, Submission on the New Zealand's International Tax Review, Developing an active income exemption for controlled foreign companies (2007).

102 Income Tax Act 2007 (NZ) s EX 22.

103 Explanatory Note, Taxation (International Taxation, Life Insurance, and Remedial Matters) Bill 2008 (NZ) p. 73.

${ }^{104}$ Explanatory Note, Taxation (International Taxation, Life Insurance, and Remedial Matters) Bill 2008 (NZ) p. 75 .
} 
que, en cualquier caso, la mayoría de ellas debería cumplir con los estándares del IFRS o el NZ-IFRS requeridos por el nuevo test de activos ${ }^{105}$.

Las pruebas o tests para determinar la calidad de no-atribuyente están sujetos a una reglamentación completa y a ajustes, de manera de asegurar que utilicen conceptos y lleguen a resultados compatibles ${ }^{106}$. En particular, el test ajustado de cuentas financieras debe ser auditado por un contador externo, quien debe proveer una 'opinión incondicional', o su equivalente en el país en el que se prepare ${ }^{107}$. Además, la Administración Tributaria no debe tener "base razonable" para dudar de dicha contabilidad o contador ${ }^{108}$.

La EEIA le otorga al contribuyente la opción de tratar diferentes SECs en la misma jurisdicción, como una SEC única, aceptando "subconsolidaciones de las SECs ubicadas en la misma jurisdicción" ${ }^{109}$, no sólo cuando las SECs son de propiedad única, como era la intención original del gobierno ${ }^{110}$, sino también cuando el contribuyente controla más del $50 \%$ de los ingresos en cada SEC, aunque en estos casos debe hacer un ajuste que refleje al interés minoritario ${ }^{111}$. No se permiten consolidaciones cuando las SEC residen en jurisdicciones diferentes, porque tal consolidación implicaría riesgos a la hacienda pública y entregaría opciones de manipulación que fueron consideradas inaceptables $^{112}$.

\subsection{Análisis}

La regla estadounidense de minimis se aplica sólo a los ingresos de Compañía Instrumental Extranjera, uno de los tipos de ingresos de la "Subsección F". Esto le impide, en principio, operar como EEIA. Además, los bajos límites numéricos y porcentuales actuales impiden que la regla opere, en los hechos, como EEIA, incluso cuando tales ingresos son los únicos que la empresa tiene. Ahora bien, dado que la regla de minimis necesariamente erosiona el principio de neutralidad de exportación de capital y posibilita oportunidades de elusión tributaria, algún beneficio, ya sea en cuanto al costo

${ }^{105}$ Explanatory Note, Taxation (International Taxation, Life Insurance, and Remedial Matters) Bill 2008 (NZ) p. 69.

106 Véase, con relación al test por defecto: Income Tax Act 2007 (NZ) s EX 21 D; y con relación al test estándar contable: Income Tax Act 2007 (NZ) ss EX 21C, EX 21E.

107 Income Tax Act 2007 (NZ) s EX 21 C (8).

108 Income Tax Act 2007 (NZ) s EX 21 C (9).

109 Gobierno de Nueva Zelanda, New Zealand's International Tax Review: Developing an active income exception for controlled foreign companies: An official's issues paper (2007), pp. 15-16.

110 "Porque de otra manera es difícil determinar la naturaleza de ingresos que deberían ser atribuidos a intereses minoritarios en subsidiarias poseídas parcialmente", Gobierno de Nueva Zelanda, New Zealand's International Tax Review: Developing an active income exception for controlled foreign companies: An official's issues paper (2007), p. 16.

111 Bajo el test por defecto, Income Tax Act 2007 (NZ) s EX 21 D (1); bajo el test de cuenta fiscal financiera ("financial account test"), Income Tax Act 2007 (NZ) s EX 21 E (2).

112 Gobierno de Nueva Zelanda, New Zealand's International Tax Review: Developing an active income exception for controlled foreign companies: An official's issues paper (2007), p. 16. 
de cumplimiento para los contribuyentes, o de fiscalización, para la Administración Tributaria, debe existir, si se quiere justificar su existencia. Lo cierto, sin embargo, es que ningún ahorro real existe, dado que el contribuyente debe hacer todos los cálculos de rutina de la "subsección F"113. Paradójicamente, entonces, la norma de minimis estadounidense es, al mismo tiempo, irrelevante y dañina: irrelevante, porque sus rigurosos límites impiden su uso efectivo como EEIA; y dañina, porque en cualquier caso, la renuncia a la neutralidad de exportación de capital se hace sin ventaja alguna como intercambio. Para entender esta situación, el desarrollo histórico de la norma es relevante. La norma original fue pensada como un instrumento que redujera los costos de cumplimiento y sirviera como EEIA. El diseño previo de la excepción de Sociedades Personales Instrumentales Extranjeras, ahora derogado, refuerza esta conclusión. Las enmiendas inorgánicas posteriores, buscando limitar el aplazamiento tributario, erosionaron estos objetivos. Sin embargo, incluso antes de las reducciones de los límites, el uso de los mismos cálculos del sistema general para determinar el test hacía que la reducción de los costos de cumplimiento fuera ilusoria.

La EEIA de Nueva Zelanda presenta la realidad opuesta: diseñada para reducir los costos de cumplimiento y para actuar como un filtro que excluya a la mayoría de las compañías del sistema de las SECs, esta norma debería tener un impacto importante no sólo en tales costos, sino también en los de fiscalización, porque el número de SECs objeto de control debería reducirse significativamente ${ }^{114}$. Por supuesto, hay un costo: bajo la EEIA, el aplazamiento tributario se expandirá y, consecuentemente, la base tributaria interna se verá erosionada. Cierto nivel de abuso parece inevitable: las compañías tendrán un incentivo importante para mantener -y manipular sus asuntos para mantener- sus pasivos bajo el umbral del $5 \%$. Sin perjuicio de este costo, el ejemplo de Nueva Zelanda parece particularmente relevante para países en desarrollo, porque concentrará la acción fiscalizadora en un número limitado, manejable, de SECs. Para países como Chile, el enfoque neozelandés, con un diseño razonable de 'gestión de riesgo', permite una fiscalización efectiva del sistema de SECs, aunque este sea un sistema tal vez más limitado y, si se quiere, más humilde que el Norteamericano.

\section{Pagos a entidades Relacionadas}

Como se postuló antes, una de las razones que explican la complejidad en el diseño y fiscalización de la normativa sobre SECs es el enorme poder y recursos de las CMN, una de las partes naturalmente interesadas en la materia. Considerando lo anterior, parece relevante discutir en forma breve una materia particular que guarda relación especial con los intereses de las CMN: el tratamiento de los desembolsos o pagos a entidades relacionadas. Este tema muestra, hasta cierto punto, los límites en la normativa sobre SECs entre cierta "conciencia de negocios" y la mera irrelevancia.

113 Arnold B.J., op. cit., p. 490.

${ }^{114}$ En 2005, 846 fueron identificadas: Explanatory Note, Taxation (International Taxation, Life Insurance, and Remedial Matters) Bill 2008 (NZ) at 75. 
Las transacciones con partes vinculadas generan un evidente riesgo para la integridad de la normativa sobre SECs ${ }^{115}$. Dos objetivos propios del sistema entran en conflicto. Por una parte, la "conciencia de negocios" demanda una limitación a la atribución, considerando que en las relaciones económicas modernas las empresas organizan sus asuntos en conglomerados que especializan sus operaciones en diferentes unidades, muchas veces, en países diferentes. Pueden existir razones legítimas de negocios -no sólo tributarias- para este tipo de organización y cualquier limitación impuesta por la normativa de SECs no sólo reduce la eficiencia del negocio, sino que, además, resta competitividad a las SECs de una empresa doméstica, frente a las SECs de una compañía extranjera, organizada de la misma manera, pero que no sufre tal atribución en su país de origen. Sin embargo, por otra parte, este tipo de pagos introduce un riesgo evidente a la base tributaria interna: la manipulación de los precios en las transacciones permite transferir las ganancias entre diferentes compañías y países. En particular, cuando las transacciones son en jurisdicciones diferentes, los riesgos son mayores y el argumento de competitividad es menos persuasivo ${ }^{116}$.

\subsection{Estados Unidos de América}

En términos generales, bajo el sistema estadounidense, el ingreso pasivo de una SEC $^{117}$ que se origina en una SEC vinculada ${ }^{118}$, es en sí mismo un ingreso pasivo. En otras palabras, en principio, bajo la normativa de las SECs de Estados Unidos, los desembolsos entre SECs son considerados ingreso "Subsección F”. Se proveen tres excepciones permanentes para aliviar esto: la excepción de minimis, ya mencionada; la excepción de tributación alta, que permite en ciertos casos evitar la atribución cuando las rentas se originan en países con alta tributación; y la excepción del mismo país. La "excepción del mismo país", en términos generales, permite que las SEC no consideren los desembolsos de dividendos e intereses de SECs vinculadas como ingreso "Subsección F" cuando la SEC vinculada, junto con una parte sustancial de sus activos, están en el mismo país ${ }^{119}$. También permite no considerar desembolsos por regalías de SECs por el uso -o el privilegio de uso- dentro del mismo país ${ }^{120}$. Esta norma está sujeta a excepciones y controles antiabusos $^{121}$. En suma, siguiendo estas reglas generales, cualquier ingreso pasivo de una SEC que se origine en otra SEC, incorporada en otra jurisdicción, vinculada o no, podría considerarse ingreso "Subsección F".

115 Arnold B.J., op. cit., p. 459.

${ }^{116}$ Ídem, p. 463.

${ }^{117}$ En particular, Ingresos de Sociedades Personales Instrumentales Extranjeras (Foreign Personal Holding Company Income), tal como son definidas en IRC s 954(c), de los cuales los más importantes están en IRC s 954 (c) (1) (A), dividendos, intereses, royalties, rentas y anualidades.

\footnotetext{
${ }^{118}$ La definición se encuentra en IRC s 954 (d)(3).

119 IRC s 954 (c)(3)(A) (i) en relación con dividendos e intereses.

${ }^{120}$ IRC s 954 (c)(3)(A)(ii) en relación con rentas y royalties.

${ }^{121}$ IRC s 954 (c)(3).
} 
Este es el sistema general permanente bajo la normativa de las SECs de EE.UU. Sin embargo, una excepción adicional temporal se sumó en $2006^{122}$. Bajo esta nueva excepción temporal, el ingreso pasivo de una SEC originado en una SEC vinculada en otra jurisdicción no será considerada “ingreso Subsección F”, siempre que el dividendo, interés, renta o regalía sean atribuibles a ingresos de la SEC pagadora que no constituyan ni ingreso "Subsección F" ni ingreso conectado de manera efectiva a la conducción de una transacción o negocio dentro de los EE.UU." ${ }^{123}$. El razonamiento detrás de esta excepción es, evidentemente, el mismo que rige a la excepción del mismo país: las CMN pueden organizarse bajo diferentes compañías para ser más eficientes y no necesariamente para evitar tributación. En el caso particular de desembolsos interjurisdiccionales, éstos pueden responder a reinversión o arreglos financieros que no deberían originar desembolsos de impuestos a accionistas estadounidenses, en especial porque estos arreglos no son objeto de tributación por otros países y, en consecuencia, hacer tributar tales arreglos colocaría a las CMN estadounidenses en desventaja para competir internacionalmente. Como es lógico, esta autorización ha debilitado en forma sustancial la normativa de las SECs Norteamericana y ha sido justamente criticada por ello ${ }^{124}$. En los Estados Unidos de Norteamérica las normas temporales tienden a transformarse en permanentes, en especial si son diseñadas para aliviar la tributación a las $\mathrm{CMN}^{125}$. Esta excepción, como se ha visto, contempla límites y reglas de protección, en particular cuando los ingresos de la SEC relacionada en definitiva provienen de Estados Unidos. El problema, sin embargo, es que cuando participan países diferentes, las posibilidades de transferir las ganancias de una jurisdicción de alta tributación a una jurisdicción con menor tributación son claras; situación ausente en el caso de desembolsos entre SECs ubicadas en el mismo país.

\subsection{Nueva Zelanda}

Como se ha señalado, bajo el sistema de SECs neozelandés previo al actual todo el ingreso de una SEC que no residía en un país de la lista gris era atribuido a los dueños neozelandeses. El nuevo sistema no sólo introdujo la EEIA, sino que limitó la atribución en el caso de SEC pasivas - esto es, SECs que no pasaban el test de EEIA- sólo al ingreso pasivo. Por ende, los desembolsos de intereses, rentas y regalías recibidas por una SEC de otra SEC, generalmente son considerados ingresos pasivos ${ }^{126}$. Sin embargo, surgió la disyuntiva de una posible excepción a la atribución cuando los desembolsos eran

\footnotetext{
122 IRC s 954 (c)(6).

123 Ídem.

${ }^{124}$ Ault H.J. y Arnold B.J., op. cit., p. 479.

$125 \mathrm{Tal}$ es el caso de excepciones relacionadas con "Ingresos Financieros Activos" ("active finance income") que ha sido prorrogado en innumerables oportunidades. Véase s 953(e)(10), 954 (h)(9).

126 Gobierno de Nueva Zelanda, New Zealand's International Tax Review: Developing an active income exception for controlled foreign companies: An official's issues paper (2007), p. 34. Las reglas para determinar los montos "Attributable" conforme a las reglas CFC: Income Tax Act 2007 (NZ) s EX $20 \mathrm{~B}$.
} 
hechos entre SEC vinculadas y activas. Resulta interesante que en la discusión se haya estudiado el régimen norteamericano ${ }^{127}$. La propuesta gubernamental fue permitir la consolidación de resultados pero sólo cuando existen pagos en la "misma jurisdicción", de manera de "evitar la tributación de ingresos activos cuando las operaciones en un país están divididas entre un número de SEC diferentes" ${ }^{28}$. La disposición norteamericana que permite consolidar los desembolsos de una compañía en "diferentes jurisdicciones" discutida en la sección anterior fue rechazada por cuanto "esas transacciones podrían llevar a transferencias ilícitas de ganancias entre jurisdicciones, de países con tributación alta a países con tributación baja"129. Durante el proceso consultivo, representantes del mundo de los negocios y asesores tributarios propusieron incorporar dicha excepción, argumentando que "los inversionistas neozelandeses en el exterior podrían establecer compañías financieras regionales" ${ }^{130}$. Sin embargo, dicha propuesta no fue considerada.

\subsection{Análisis}

El problema de los pagos entre partes relacionadas muestra las contradicciones inherentes a las políticas tributarias en que se sustenta la normativa SEC. Por un lado, la neutralidad en la importación de capital requiere aceptar dichos desembolsos. No existe una razón a priori para ofrecer alivio en el caso de desembolsos en la misma jurisdicción y negarlo cuando son distintas. Organizaciones legítimas de negocios, en particular aquellas vinculadas con gestión financiera regional, pueden requerir de organizaciones en jurisdicciones diferentes. Por ejemplo, un conglomerado que opera en Sudamérica podría utilizar a Chile como su oficina central regional, por sus relativamente sofisticados sistemas bancarios, de comunicaciones y de regulación comercial, proporcionando gestión y servicios financieros a todas las operaciones del área. Si cada una de dichas operaciones constituyen SECs activas, en principio no existen razones para no considerar el esquema completo como un negocio activo. Sin embargo, por el otro lado, dicho esquema puede utilizarse fácilmente para transferir ganancias entre jurisdicciones y manipular los tributos. Los impuestos son, en términos comerciales, un gasto más y, evidentemente, un esquema como éste se utilizaría para reducir la carga tributaria lo más posible, haciendo irrelevante la figura de las SEC.

Se puede argumentar que la disposición de consolidación norteamericana pone a ese sistema - una vez más- en el peor de los casos. El sistema norteamericano sobre SECs es extraordinariamente complejo, con altos costos de fiscalización para la Administración Tributaria, y significativos costos de cumplimiento para los contribuyentes. Estos costos,

127 Gobierno de Nueva Zelanda, New Zealand's International Tax Review: Developing an active income exception for controlled foreign companies: An official's issues paper (2007), pp. 35-36.

128 Ídem, p. 36.

129 Ídem.

${ }^{130}$ New Zealand Institute of Chartered Accountants, Submission on the Government Officials' issues paper: New Zealand's International Tax Review, Developing an active income exemption for controlled foreign companies (2007), p. 21. 
sin embargo, son menos significativos para las grandes CMN, entes que por definición tienen ingresos en distintas jurisdicciones, las que pasan a contar con una herramienta adicional para rebajar su carga tributaria. Es el peor de los casos porque un número mayor de contribuyentes - los pequeños- pierden, pero aquellas empresas que son el objetivo principal de la normativa sobre SECs, las CMN, resultan beneficiadas. En este ámbito, el enfoque neozelandés parece razonable. En una economía moderna, los desembolsos entre SEC vinculadas en la misma jurisdicción son normales y otorgan oportunidades limitadas de manipulación.

\section{Conclusiones}

Doscientos años después que Francisco de Goya creara su famoso grabado, "el sueño de la razón produce monstruos”; los eruditos discuten aún su significado. En el cuadro, un hombre -probablemente el mismo artista- duerme con su cabeza sobre una mesa, rodeado de búhos y murciélagos. Una posible interpretación es que, cuando la razón es suprimida y la imaginación, los sueños e ideales se desatan el resultado es la locura y la ignorancia. Parte del genio del artista, por supuesto, es que es posible la interpretación exactamente opuesta: una razón ilimitada -el sueño de esa razón- produce tales males. Tal fue la contradicción inherente de su época, entre la ilustración y el romanticismo, y el genio del artista al mostrárnosla.

Este trabajo ha buscado compartir un análisis comparativo de las normativas referentes a las SECs, una materia extraordinariamente compleja, tratando de identificar elementos que deberían ser considerados, en particular por los países en desarrollo, a la hora de diseñar un sistema SEC relevante, razonable y fiscalizable. Las normas sobre SECs establecidas para materializar políticas inherentemente contradictorias deben seguir un enfoque realista y razonable, porque tienen el potencial de volverse inmanejables, costosas e incomprensibles. Un enfoque "unilateral" en cualquiera de las materias discutidas: un cuidado extremo en cubrir todas las posibilidades en la definición de las SEC, las limitaciones en el diseño de las EEIA para evitar cualquier erosión en la base doméstica o una lógica comercial excesiva en lo que respecta a los pagos entre partes relacionadas, lleva a soluciones insatisfactorias. Sólo una consideración equilibrada de las políticas y valores que conciernen al diseño de las SECs, sin dejarse llevar por los sueños de la razón -o del idealismo-, puede evitar sus monstruos.

\section{BIBLIOGRAFÍA}

ARnold, B.J., The Taxation of Controlled Foreign Corporations: an International Comparison, Canadian Tax Fundation, Toronto, 1986.

Arnold, J.A. y Dibout, P., 'Limits on the use of low-tax regimes by multinational business: current measures and emerging trends. General Report', en Cabiers de Droit Fiscal International, LXXXVIb, 2001. 
Ault, H.J. y Arnold, B.J., Comparative Income Taxation: A Structural Analysis (Tercera Edición) KluwerLaw International, La Haya, 2010.

Ault, H.J., "Tax Simplification from a Comparative Point of View”, en Lindencrona G, Lodin S. y Wiman B. (editores), International Studies in Taxation: Law and Economics, Kluwer Law International, La Haya, 1999.

Biastrocchi, E., 'The use and Interpretation of Tax Treaties in the emerging world: Theory and Implications', en British Tax Review, 4, 2008.

Burns, L., Controlled Foreign Companies: Taxation of Foreign Source Income, Longman Professional, Melbourne, 1992.

Business New Zealand, Submission on the New Zealand's International Tax Review, Developing an active income exemption for controlled foreign companies (2007) < http://www.businessnz.org.nz/ submissions?page $=10>$, fecha de revision, 21-05-2010.

Dryzer, J.S., Deliberative Democracy and Beyond: Liberals, Critics, Contestations, Oxford University Press, Nueva York, 2002.

Freedman, J., Loomer, G. y Vella, J., 'Corporate Tax Risk and Tax Avoidance: New Approaches', en British Tax Review, 1, 2009.

Gobierno de Nueva Zelanda, New Zealand's International Tax Review: A direction for Change, 2006. El documento puede ser consultado en la página web de la Inland Revenue de Nueva Zelanda, <http://taxpolicy.ird.govt.nz/publications/year/2006>, fecha de revisión, 30-082010 .

Gobierno de Nueva Zelanda, New Zealand's International Tax Review: Developing an active income exception for controlled foreign companies: An official's issues paper (2007). El documento puede ser consultado en la página web de la Inland Revenue de Nueva Zelanda, <http://taxpolicy. ird.govt.nz/publications/year/2007 >, fecha de revisión, 30-08-2010.

Gobierno de Nueva Zelanda, Explanatory Note, Taxation (International Taxation, Life Insurance, and Remedial Matters) Bill 2008 (NZ). El texto íntegro del Bill puede ser consultado en la página del parlamento de Nueva Zelanda, <http://www.parliament.nz/en-NZ/PB/Legislation/ Bills/e/0/e/00DBHOH_BILL8642_1-Taxation-International-Taxation-Life-Insurance.htm>, fecha de revisión, 30-08-2010; o bien en la página de la Inland Revenue del mismo país, $<$ http://taxpolicy.ird.govt.nz/publications/2008-bill-itlirm/overview >, fecha de revisión, 30-08-2010.

Isenbergh, J., International Taxation (Segunda Edición), Fundation Press, Nueva York, 2005.

Jones, J.A. 'Tax Law: Rules or Principles?', British Tax Review, 6, 1996.

McDaniel, P.R, Ault, J.A. y RepetTi, J.R., Introduction to United States International Taxation (Quinta edición), Kluwer Law International, La Haya, 2005.

New Zealand Master Tax Guide, Wolters Kluwer Business, Auckland, 2010.

New Zealand Institute of Chartered Accountants, Submission on the Government Officials' issues paper: New Zealand's International Tax Review, Developing anactive income exemption for controlled foreign companies $(2007)<\mathrm{http}: / /$ www.nzica.com/AM/Template.cfm?Section =Tax_ Submissions_Files\&Template $=/ C M /$ ContentDisplay.cfm\&ContentID $=10279>$ fecha de revision, 21-05-2010.

Vann, R.J., "International Aspects of Income Tax", en Thuronyi V. (editor), Tax Law Design and Drafting, International Monetary Fund, Washington, 1996. 